\title{
Problematização aplicada ao ensino e aprendizagem de algoritmos: Uma análise dos fatores associados a motivação dos estudantes
}

\author{
Evandro Franzen - UNIVATES/ PPGIE UFRGS - evandrofra@gmail.com \\ Magda Bercht - PPGIE UFRGS - bercht@inf.ufrgs.br \\ Juliano Dertzbacher-UNIVATES - juliano.dertzbacher@univates.br
}

\begin{abstract}
Resumo. Este artigo apresenta uma investigação sobre a viabilidade da observação e identificação de fatores que indiquem a motivação dos estudantes no processo de ensino e aprendizagem de algoritmos. A estratégia de ensino utilizada é baseada na problematização com o Arco de Maguerez, e consiste no desenvolvimento de atividades que estimulem o aluno a compreender e descrever o problema antes da elaboração do código fonte. $\mathrm{O}$ experimento descrito envolveu 14 estudantes, em um estudo de caso, realizado na disciplina de Algoritmos e Programação. Durante o desenvolvimento das atividades foram coletados dados dos logs do ambiente virtual, que viabilizaram a elaboração de hipóteses quanto ao esforço, confiança e independência demonstradas. Foi possível perceber que os estudantes nem sempre se dedicam da maneira esperada e em alguns casos demonstram dificuldade para formalizar detalhadamente o problema. Os resultados demonstraram que a identificação dos fatores relacionados à motivação com o uso problematização, é viável.
\end{abstract}

Palavras-chave: Ensino e aprendizagem de algoritmos, motivação, problematização.

\section{Problematization applied to teach and learn algorithms: An analysis of the factors associated with student motivation}

Abstract. This paper presents an investigation about viability of observing and identifying factors that indicate the students' motivation in the process of teaching and learning algorithms. The teaching strategy used is based on the problematization with the Arch of Maguerez and consists in the activities development that stimulate the student to understand and describe the problem before the source code elaboration. The experiment described involved 14 students, in a case study, carried out in the discipline of Algorithms and Programming. During the activities, development data were collected from the logs of the virtual environment, which enabled the hypotheses elaboration regarding effort, confidence and independence demonstrated. It was possible to perceive that the students do not always dedicate themselves in expected way and in some cases they demonstrate difficulty to formalize the problem in detail. The results demonstrated that the identification of motivation factors related to with problematization use, is feasible.

Keywords: Teach and learn algorithms, motivation, problematization.

\section{Introdução}

As disciplinas de algoritmos e programação são fundamentais nos cursos da área de computação. Estudos demonstram que há uma grande taxa de insucesso nas disciplinas desta área. Watson e $\mathrm{Li}$ (2014) revisando o trabalho desenvolvido por Bennedsen e Caspersen (2007) demonstram que a média de reprovação é superior a 30\%, ou seja, aproximadamente um terço dos alunos é reprovado. O cenário brasileiro pode ser ainda pior, Ramos (2015) apresenta resultados estimados que indicam taxas de reprovações que podem variar de 40 a 50\% em instituições nacionais.

A maioria dos cursos de graduação na área de computação, no Brasil, são concebidos com base nas diretrizes nacionais curriculares (MEC, 2016). Entre as competências e habilidades a serem desenvolvidas nestas disciplinas, estão a identificação de problemas que tenham solução algorítmica e a resolução dos mesmos 
usando algoritmos e ambientes de programação. O que se observa é que grande parte dos alunos apresenta dificuldades relacionadas à abstração, à compreensão do problema, das estruturas, e da sintaxe da linguagem. Estudo desenvolvido por Da Costa Mora e Giraffa (2016) mostra que grande parte dos alunos indica que tem dificuldade de compreender o enunciado e o que é solicitado, mas ao mesmo tempo reconhece que não faz perguntas ou questionamentos para resolução de dúvidas.

A elaboração de algoritmos pressupõe uma capacidade de resolver problemas utilizando uma sequência de passos que compõe uma solução. Tradicionalmente o ensino se dá pelo desenvolvimento de exercícios a partir de enunciados que apresentam problemas a serem resolvidos pelo aluno (Iepsen, 2013). Antes da codificação e da tradução para uma linguagem de máquina, é importante que este compreenda o problema e defina uma estrutura lógica da solução, além dos elementos que serão usados nele (estruturas de armazenamento, condicionais, repetição, entre outros).

A falha na identificação destes elementos pode dificultar a busca pela solução e em alguns casos levar a um cenário de desmotivação. A maioria dos estudantes têm dificuldades ou não é estimulado a compreender e formalizar problemas antes da construção da solução. Além disso, boa parte dos professores não possui informações sobre a maneira utilizada pelos alunos para entender o problema e estruturar a solução. A partir destas constatações buscou-se uma abordagem que estimulasse esta reflexão e permitisse analisar o comportamento dos estudantes após a realização das atividades. Optou-se pela utilização de uma estratégia baseada na problematização, que que privilegia a compreensão e elaboração de hipótese para resolução de um problema antes da elaboração e aplicação de uma solução desenvolvida (Berbel, 2011).

Metodologias de ensino ativas, como a problematização, têm sido utilizadas com o objetivo de estimular o envolvimento, colocar o discente como sujeito fundamental no processo de ensino e aprendizagem. Para que o envolvimento e a participação ativa dos estudantes ocorra é essencial que estes reconheçam a importância das atividades e estejam motivados para realizar as ações requeridas. Uma análise da trajetória do estudante, como este realiza as atividades, a sequência, os tempos, o acesso aos recursos de ajuda, e os conteúdos das respostas em cada etapa pode indicar a viabilidade da observação de fatores associados a motivação apresentada pelos discentes.

O experimento apresentado neste trabalho, foi realizado em uma disciplina introdutória de algoritmos e programação. Na seção 2 serão apresentados os pressupostos teóricos nos quais se baseia o trabalho. A seguir são descritos $o$ experimento realizado e os principais resultados, com ênfase nos dados coletados e nos apontamentos feitos a partir da pesquisa sobre a percepção dos estudantes. Conclusões preliminares são descritas na seção 6 .

\section{Fundamentação}

As metodologias de ensino e aprendizagem ativas constituem-se em abordagens que estimulam o envolvimento e a participação ativa dos estudantes no processo, desenvolvendo a autonomia e a capacidade de reflexão sobre as situações vivenciadas. Segundo Berbel (2011) estas contribuem para aumentar a curiosidade e motivação, uma vez que aproximam o estudante da realidade, fortalecendo a percepção sobre a importância da compreensão e reflexão sobre os problemas apresentados.

Diversas técnicas ou metodologias que podem ser classificadas como ativas, entre elas a problematização, que teve como um dos primeiros métodos propostos, o Arco de Maguerez (Figura 1). Uma das referências iniciais a esta metodologia é encontrada em Bordenave e Pereira (1982), nela enfatiza-se a ideia de que a aprendizagem deve acontecer a partir de uma visão global de um problema para uma 
visão analítica, que equivale a compreensão do mesmo. A situação problema diz respeito à realidade ou a um recorte desta, sendo possível abordar desde problemas mais complexos aos mais simples, de acordo com a proposta do estudo. O problema é definido a partir da observação da realidade ou da contextualização desta por parte do professor em conjunto com os estudantes.

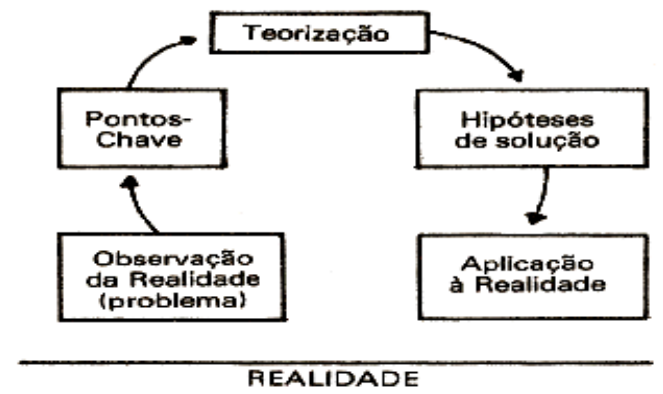

Figura 1 - Arco de Maguerez (Bordenave e Pereira, 1982)

A definição dos pontos chave do problema permite uma compreensão inicial dos principais elementos que o definem. Estabelece-se neste momento uma reflexão sobre o problema, a determinação da sua complexidade e as variáveis associadas. Busca-se identificar quais conceitos devem ser pesquisados ou revisados para a proposição de alternativas que possam solucioná-lo (Berbel, 2012).

A reflexão e estudos devem fornecer subsídios para a elaboração de possíveis soluções, ou seja, hipóteses de solução para o problema. Quais recursos serão utilizados, quais sequências de passos são adequadas para elaborar a solução, que técnicas serão usadas para elaborar, testar e validar a solução, são exemplos de questionamentos comuns nesta etapa (Berbel, 2011). A discussão e interação entre os participantes e professor é estimulada especialmente nesta etapa, momento no qual as diferentes hipóteses são analisadas.

A motivação do aluno é condição essencial para desenvolver a autonomia e a capacidade de tomar decisões durante o processo de resolução de problemas (Berbel, 2012). O uso da problematização tem como objetivo motivar os discentes, entretanto, não é possível garantir que a simples utilização do método é suficiente para atingir este objetivo. Um acompanhamento das ações dos estudantes, da forma que estes problematizam, da sua trajetória durante o processo pode ser importante para avaliar os resultados obtidos. Uma das abordagens que pode ser usada para observar e a analisar os sujeitos durante as etapas da problematização, é a que considera a dimensão afetiva, ou, os estados emocionais.

O estado emocional ou estado mental afetivo diz respeito ao conjunto de características que determinam a emoção em um indivíduo, em determinado momento Emoções podem ser caracterizadas como reações expressivas e fisiológicas, como sentimentos ou comportamentos diante de determinadas situações (Bercht, 2001). Entre os diversos estados afetivos, pode-se citar frustração, interesse e a motivação. As emoções ou sentimentos podem influenciar direta ou indiretamente o processo de aprendizado.

A Computação Afetiva é a área da computação que investiga os fundamentos e aplicações que visam capacitar os computadores para identificar, compreender e representar estados afetivos (Picard 1997). Conferir a um sistema recursos para identificar que um estudante se encontra desmotivado, por exemplo, pode permitir a indicação de ações ou tarefas alternativas, durante a resolução de um problema.

Embora considerados muitas vezes sinônimos, motivação, interesse ou engajamento não são caracterizados pelo mesmo estado mental e a identificação de cada 
um envolve diferentes estratégias de observação e coleta de dados. Utilizar sistemas computacionais que capturem informações sobre o comportamento do aluno, como o número de erros, tempo de dedicado, a um mesmo exercício ou o número de tentativas é uma abordagem comum (Longhi, Behar, Bercht, 2010; Iepsen, 2013). Estratégias mistas combinam indicadores oriundos de coletas automáticas com informações observadas e registradas manualmente por um professor ou pesquisador.

O foco principal deste trabalho é na motivação e nos princípios e modelos usados para reconhecer este estado afetivo. Diversos modelos têm sido propostos para definir e identificar a motivação, entre eles o modelo Esforço (E), Independência (I) e Confiança (C) (EIC), apresentado em (Del Soldato e Du Boulay, 1995). Uma revisão do modelo foi descrita pelos autores em (Du Boulay e Del Soldato, 2016). A motivação é definida de forma pragmática, em uma combinação do esforço empreendido para o aprendizado, da confiança que o aluno demonstra em sua habilidade de aprender ou resolver determinada situação, com um mínimo de auxílio o que caracteriza um comportamento mais independente.

$\mathrm{Na}$ visão dos autores o esforço pode ser medido a partir da dedicação e perseverança do estudante, de uma análise de como ele enfrenta dificuldades e obstáculos. A confiança e a independência podem ser obtidas pela observação da quantidade de auxílio demandada durante a atividade. Alunos desmotivados na maioria das situações não se esforçam ou não acreditam que podem ter sucesso na resolução das tarefas (Du Boulay e Del Soldato, 2016).

\section{Trabalhos relacionados}

Um dos trabalhos que servem de referência para este estudo foi desenvolvido por Iepsen (2013). Nele são utilizadas técnicas de mineração de dados baseadas em regras para encontrar padrões de comportamento associados a frustração no aprendizado de algoritmos. São coletados dados relativos à número de compilações com erros, além dos tempos para a elaboração do código fonte. É apresentado um sistema utilizado pelos alunos para escrever e compilar o algoritmo e enquanto estes resolvem a questão, têm a possibilidade de indicar se está frustrado clicando em um botão disponível no software. Posteriormente o processo de mineração encontra regras que relacionam alto número de compilações ou a incidência recorrente de erros em programas com o estado de frustração indicado pelo usuário.

Buscando complementar o trabalho de Iepsen (2013) o experimento descrito neste artigo propõe coleta e análise de dados nas etapas anteriores a escrita do código fonte. A análise de dados durante as etapas de problematização, não apenas na escrita do código pode contribuir para uma percepção mais completa sobre o estado de ânimo do estudante.

A pesquisa de Cocea e Wiebelzahl (2011) descreve uma análise de indicadores de engajamento a partir de dados coletados em um ambiente denominado HTML-Tutor utilizado por alunos em um curso de HTML. Foram coletadas informações sobre diversos atributos, incluindo testes executados, leitura de páginas, buscas, acesso a hiperlinks e glossários. A utilização de redes bayesianas para reconhecer estados de ânimo é apresentada por Longhi, Behar e Bercht (2010). No referido estudo foram definidas variáveis relacionadas ao comportamento observável, entre estes o número de acessos, participação em fóruns, frequência, pedidos de ajuda e o tempo de permanência em cada sessão.

A coleta de dados e proposição de variáveis relacionadas a estados de ânimo é uma característica comum entre os trabalhos citados. A análise destes serviu de base para a definição das variáveis utilizadas neste estudo, como, por exemplo, informações 
relativas aos acessos do estudante e aos tempos demandados para as diversas atividades. Busca-se no presente trabalho, complementar os estudos apresentados, associando os fatores da motivação a uma metodologia de ensino e aprendizagem ativa, que busca a reflexão e compreensão dos problemas antes da elaboração da solução final.

\section{Materiais e métodos}

Este trabalho apresenta uma pesquisa de natureza exploratória, um estudo de caso realizado com alunos de uma disciplina de Algoritmos e Programação, no qual investigou-se a possibilidade de utilizar uma estratégia de ensino baseada na problematização com o Arco de Maguerez (Berbel, 2011). Durante as atividades foram coletados dados com o objetivo de analisar a possibilidade de identificar fatores associados a motivação dos estudantes. Com o objetivo de complementar a análise dos dados obtidos nos logs disponíveis no ambiente, foi aplicado um questionário para que o estudante relatasse a sua percepção sobre as atividades desenvolvidas.

A disciplina ocorreu na modalidade presencial e contou com a participação de 14 estudantes. Todas as atividades ocorreram em um laboratório de informática, com a alocação de um computador por aluno, fator que facilita uma orientação e acompanhamento mais direto e próximo por parte do professor. $\mathrm{O}$ cenário exposto contribuiu para a pesquisa sobre métodos que busquem a reflexão sobre problemas e que demandem uma análise detalhada do comportamento dos estudantes, durante o desenvolvimento das tarefas. O Ambiente Virtual de Ensino e Aprendizagem (AVEA) Moodle foi usado como plataforma de apoio e coleta de dados. O recurso denominado Lição foi usado para implementar atividades seguindo as etapas mostradas na Figura 2.

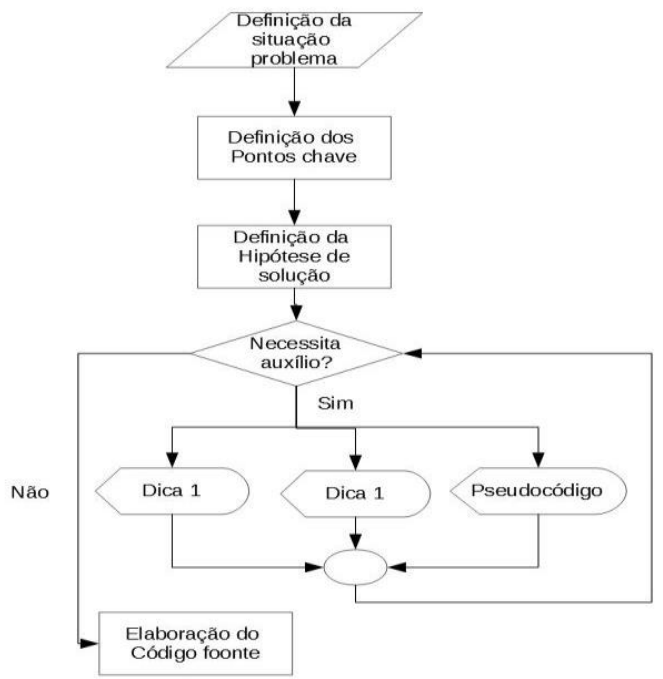

Figura 2 - Fluxo de atividades

$\mathrm{Na}$ definição dos pontos chave o estudante foi estimulado a compreender e pensar nos recursos e elementos que usaria para solucionar o problema. A hipótese de solução consistiu na definição de uma sequência de passos, blocos, indicando uma solução em alto nível, sem a utilização de instruções de programação, como ocorreu na codificação. Dicas e a listagem do próprio pseudocódigo foram oferecidas como forma de ajuda para a tarefa. O acesso a este conteúdo era opcional, a decisão de buscar auxílio foi delegada ao próprio aluno.

Foram propostos 8 exercícios de programação, iniciando por questões simples, relacionadas à entradas, saídas e atribuições, culminando por atividades que requeriam o uso de estruturas mais complexas como matrizes. Diferentemente dos enunciados clássicos que indicam ao aluno ações, como "ler duas variáveis inteiras", "calcular a 
divisão de x e y", "ler uma matriz de 5x5", a descrição do problema apresentava uma situação real, para a qual seria construído um programa de computador. A disponibilização de recursos que auxiliam o aluno e o incremento na complexidade do problema, seguem a estratégia proposta por (Del Soldato e Du Boulay, 1995)

Após o período de realização das atividades, foi aplicado questionário que teve como objetivo coletar dados relacionados à percepção que os alunos tiveram sobre as atividades. A natureza das questões e os resultados são apresentados na seção 4.2.

\section{Resultados e discussão}

Inicialmente os registros obtidos no log do AVEA Moodle (Tabela 1), foram analisados com objetivo de verificar a relevância de cada ação e quais informações poderiam ser obtidas. A data e a hora de cada uma das ações, bem como as páginas e recursos acessados, constituíram-se em dados essenciais para definição das variáveis propostas.

Tabela 1 - Exemplos de registros de log coletados

\begin{tabular}{|l|l|l|}
\hline Data/hora & Ação & Descrição \\
\hline 10/11/2016 19:20 & Lição iniciada & The user with id '771' started the lesson with course module id '558104'. \\
\hline 10/11/2016 19:20 & Pergunta visualizada & $\begin{array}{l}\text { The user with id '771' has viewed the Dissertação question with id '7188' } \\
\text { in the lesson activity with course module id '558104'. }\end{array}$ \\
\hline 10/11/2016 19:24 & Pergunta respondida & $\begin{array}{l}\text { The user with id '771' has answered the Dissertação question with id } \\
\text { '7188' in the lesson activity with course module id '558104'. }\end{array}$ \\
\hline
\end{tabular}

Levando em consideração os modelos encontrados em (Du Boulay e Del Soldato, 2016; Bercht, 2001; Longhi e Bercht, 2010), foram propostas variáveis relacionadas ao esforço (tempos, nível de detalhe), confiança e independência (acesso as dicas, retomadas e o acesso anterior à realização da tarefa). As principais variáveis derivadas do log são:

- Tempos: Tempo para descrição dos pontos chave do problema, tempo para descrição da proposta ou hipótese de solução, tempo para a escrita do código fonte e o tempo total da atividade.

- Acesso às dicas: Indica se acessou o texto das duas dicas e do pseudocódigo.

- Número de retomadas: Quantas vezes o aluno abandonou e retomou a atividade.

- Acesso anterior: Indica se acessou em determinada data e retornou posteriormente, em outro momento para resolver as questões.

Tabela 2 - Respostas com alto e baixo nível de detalhamento

\begin{tabular}{|l|l|l|}
\hline Questão & Baixo detalhamento & Alto detalhamento \\
\hline $\begin{array}{l}\text { Pontos } \\
\text { chave }\end{array}$ & $\begin{array}{l}\text {-Variaveis } \\
\text {-entradas } \\
\text {-matriz }\end{array}$ & $\begin{array}{l}\text { criar variáveis inteiras: uma matriz de inteiros } \\
\text { uma entrada para a matriz, para saber o número de linhas } \\
\text { uma entrada para armazenar o tipo } \\
\text { laço condicional; laço de repetição } \\
\text { uma entrada para pedir o tipo armazenado; imprimir o pedido }\end{array}$ \\
\hline Hipótese & $\begin{array}{l}\text { matriz que quando o } \\
\text { usuário indicar tais } \\
\text { posiçoes salvar a a a } \\
\text { entrada }\end{array}$ & $\begin{array}{l}\text { Gerar matriz equivalente a prateleira 12x3; gerar variaveis lin col } \\
\text { fazer dois laços de repetiçao para preencher a matriz } \\
\text { apos preenchida a matriz fazer testes com if para analisar quantos produtos } \\
\text { de cada tipo existem em cada prateleira; fechar laços } \\
\text { pedir ao usuario qual prateleira ele deseja; dois laços de repetiçao } \\
\text { informar os valores de tal prateleira; fechar laços }\end{array}$ \\
\hline
\end{tabular}

A análise do conteúdo das respostas para as duas primeiras questões redundou em duas variáveis que indicam o nível de detalhamento (alto, baixo ou médio) que corresponde a completude e o tamanho da resposta, tanto para os pontos chave, quanto para a hipótese de solução. O nível de detalhamento nas duas questões é importante para indicar um maior ou menor grau de esforço na resolução da atividade. No caso da identificação dos pontos chaves do problema, em alguns casos verificou-se respostas 
que incluíam todas ou maioria das variáveis, com os tipos correspondentes e outras respostas que apenas citavam como ponto chave "variáveis" (Tabela 2).

$\mathrm{Na}$ definição de uma hipótese ou especificação da solução, um maior detalhamento pode permitir que outra pessoa produza a solução, além de demonstrar a capacidade do estudante para analisar o problema e estruturar uma solução antes de iniciar a escrita do código fonte.

\subsection{Análise dos dados coletados}

Foram obtidos registros de 105 atividades, realizadas pelos alunos. A Tabela 3 apresenta informações agrupadas por estudante. Os nomes foram omitidos e definidos como um identificador sequencial.

Tabela 3 - Tempos de resolução das tarefas por aluno

\begin{tabular}{|c|c|c|c|c|c|c|c|c|}
\hline Aluno & \multicolumn{7}{|c|}{ Tempo médio e desvio padrão (minutos) } & \multicolumn{2}{c|}{ Proporção de tempo médio } \\
\hline & \multicolumn{2}{|c|}{ Pontos chave } & \multicolumn{2}{c|}{ Hipótese } & \multicolumn{2}{c|}{ Código fonte } & Pontos chave + Hipótese & Código fonte \\
\hline 1 & 10,00 & 8,88 & 3,50 & 2,69 & 27,83 & 30,52 & $32,27 \%$ & $67,73 \%$ \\
\hline 2 & 7,00 & 5,45 & 6,50 & 6,42 & 23,75 & 14,62 & $36,24 \%$ & $63,76 \%$ \\
\hline 3 & 17,25 & 11,11 & 4,75 & 6,60 & 21,12 & 24,04 & $51,01 \%$ & $48,99 \%$ \\
\hline 4 & 16,37 & 14,29 & 8,00 & 4,72 & 13,25 & 15,51 & $64,78 \%$ & $35,22 \%$ \\
\hline 5 & 30,62 & 30,66 & 24,12 & 25,50 & 28,00 & 23,92 & $66,16 \%$ & $33,84 \%$ \\
\hline 6 & 6,12 & 4,05 & 4,00 & 3,50 & 23,87 & 16,40 & $29,78 \%$ & $70,22 \%$ \\
\hline 7 & 13,62 & 6,09 & 10,75 & 6,51 & 18,62 & 18,07 & $56,69 \%$ & $43,31 \%$ \\
\hline 8 & 18,75 & 19,46 & 3,50 & 2,67 & 24,00 & 18,96 & $49,73 \%$ & $51,89 \%$ \\
\hline 9 & 9,50 & 9,98 & 5,87 & 7,58 & 26,25 & 20,23 & $36,94 \%$ & $63,06 \%$ \\
\hline 10 & 17,12 & 24,19 & 3,50 & 3,81 & 23,50 & 19,06 & $47,19 \%$ & $52,81 \%$ \\
\hline 11 & 30,00 & 28,01 & 11,75 & 13,25 & 12,25 & 15,34 & $76,96 \%$ & $23,04 \%$ \\
\hline 12 & 9,62 & 10,54 & 4,12 & 4,54 & 25,00 & 30,56 & $35,48 \%$ & $64,52 \%$ \\
\hline 13 & 19,57 & 17,43 & 9,71 & 6,75 & 45,42 & 46,81 & $39,20 \%$ & $60,80 \%$ \\
\hline 14 & 8,62 & 3,99 & 9,75 & 6,29 & 29,37 & 24,34 & $35,00 \%$ & $65,00 \%$ \\
\hline
\end{tabular}

A média e o desvio padrão para cada estudante foi calculada tendo como base todas as atividades realizadas ao longo do experimento. Observa-se uma grande variação no tempo médio dedicado à cada uma das tarefas, enquanto alguns apresentam médias altas, entre 20 e 30 minutos para a definição dos pontos chave, outros utilizam menos da metade do tempo para resolver a questão.

Descrever uma hipótese de solução normalmente exige uma compreensão mais profunda do problema e uma reflexão (Berbel, 2011), entretanto, o tempo dedicado à primeira etapa é quase sempre superior à segunda e em alguns casos é mais do que o dobro. Observou-se que muitas vezes a estratégia adotada foi identificar os pontos chave e no mesmo momento estruturar um esboço da solução e só depois postar os conteúdos para as duas respostas no ambiente. Valores muito reduzidos podem indicar pouco esforço ou que o aluno não considera importante compreender e formalizar o problema antes de elaborar a solução. Estabelece-se aqui uma analogia dos tempos identificados com o número de tentativas e medidas de perseverança encontradas em Del Soldato Du Boulay (1995).

Os valores do desvio padrão mostrado indicam que há discrepâncias em relação à média. Há uma grande variação entre o tempo dedicado em um exercício e em outro, na mesma etapa. Pode-se citar o exemplo de estudantes que dedicaram 2 minutos para a elaboração do código fonte em determinada atividade e em outras, tempos superiores a uma hora. Tempos muito reduzidos em algumas etapas, principalmente, na escrita do código fonte, geraram questionamentos sobre a possibilidade do aluno ter copiado de forma completa ou parcial uma solução de alguma fonte ou de um colega. Este questionamento decorre também do fato de que o código não é produzido de forma integrada no ambiente, sendo apenas postado pelo estudante quando finalizado. 
Na maioria das atividades é despendido um tempo maior para a escrita do código fonte. Entretanto, em alguns casos a soma do tempo das duas primeiras atividades representa mais de $60 \%$ do tempo total e em um dos casos, é superior a $70 \%$. Analisando os dados da Tabela 4, observa-se que estudantes que apresentam tempos muito maiores nas duas primeiras etapas costumam acessar frequentemente as dicas ou param e retomam a atividade mais vezes. Uma hipótese possível é que este comportamento pode estar associado à baixos níveis de confiança e independência, além de indicar uma certa dificuldade na compreensão do problema.

A predominância por níveis baixos ou médios no detalhamento do problema é visível (Tabela 4). Não é possível estabelecer uma relação direta entre o tempo dedicado e o nível de detalhamento das questões iniciais. Tempos médios altos nas primeiras etapas, como nos exemplos dos alunos 4 e 5 , não resultam necessariamente em maior detalhamento nas respostas. Ao analisar os conteúdos das respostas dos estudantes verifica-se que as mesmas apresentam muitas semelhanças nas duas primeiras etapas e, em alguns casos, são praticamente iguais. Tal fato também contribui para uma redução dos tempos destinados a descrição da proposta de solução.

Tabela 4 - Estatísticas sobre acessos e detalhamento por aluno

\begin{tabular}{|c|c|c|c|c|c|c|c|c|c|}
\hline Aluno & Número de & Número de & \multirow{2}{*}{$\begin{array}{c}\text { Acesso } \\
\text { acesso às } \\
\text { dicas }\end{array}$} & retomadas & \multicolumn{6}{|c|}{ Nível de detalhamento } \\
\cline { 5 - 10 } & & & & & \multicolumn{3}{|c|}{ Hontos chave } & \multicolumn{4}{|c|}{ Hipótese } \\
\hline & & & & Alto & Médio & Baixo & Alto & Médio & Baixo \\
\hline 1 & 4 & 2 & 1 & 0 & 0 & 6 & 0 & 4 & 2 \\
\hline 2 & 0 & 4 & 2 & 0 & 3 & 4 & 4 & 3 & 1 \\
\hline 3 & 0 & 5 & 3 & 1 & 3 & 4 & 1 & 5 & 2 \\
\hline 4 & 11 & 17 & 5 & 0 & 3 & 5 & 1 & 3 & 4 \\
\hline 5 & 5 & 21 & 6 & 2 & 3 & 3 & 3 & 4 & 1 \\
\hline 6 & 3 & 6 & 2 & 0 & 6 & 2 & 0 & 6 & 2 \\
\hline 7 & 7 & 17 & 5 & 1 & 0 & 7 & 3 & 4 & 1 \\
\hline 8 & 9 & 9 & 1 & 1 & 7 & 0 & 1 & 5 & 2 \\
\hline 9 & 0 & 3 & 0 & 5 & 2 & 0 & 0 & 4 & 4 \\
\hline 10 & 6 & 7 & 2 & 1 & 5 & 2 & 0 & 4 & 4 \\
\hline 11 & 8 & 11 & 0 & 0 & 4 & 0 & 0 & 4 & 0 \\
\hline 12 & 13 & 6 & 2 & 0 & 2 & 6 & 0 & 1 & 7 \\
\hline 13 & 7 & 7 & 3 & 2 & 2 & 3 & 1 & 7 & 0 \\
\hline 14 & 1 & 4 & 1 & 0 & 3 & 5 & 0 & 5 & 3 \\
\hline
\end{tabular}

O número de vezes que o aluno interrompe e atividade e retoma posteriormente, combinado com os acessos realizados em uma data anterior podem ser usados como indicativos para a confiança e independência. É possível constatar que aqueles que acessam constantemente as dicas também apresentam vários acessos anteriores, ao contrário dos estudantes que pouco utilizam as dicas.

\subsection{Avaliação da percepção dos estudantes}

A última etapa do experimento foi a aplicação de um questionário para que os estudantes avaliassem a atividade e fizessem um auto relato sobre a forma de resolver o problema e a dedicação à resolução dos problemas. Foram elaboradas 13 questões sobre a visão do aluno sobre a problematização, a forma de representação usada, a percepção sobre a sua dedicação e esforço, o acesso às dicas e os motivos que o fizeram acessar ou não e por último a satisfação geral e percepção sobre a resolução de problemas.

Em sua totalidade, os estudantes entendem que compreender o problema antes de escrever o código contribui parcialmente (47\%) ou integralmente $(53 \%)$ para melhorar o desenvolvimento da solução final, mas um terço acredita que é mais fácil escrever o código antes de descrever o problema. Constatou-se que $60 \%$ indicou que 
compreendeu parcialmente o problema, enquanto $26,70 \%$ indicam que compreenderam integralmente o que era solicitado.

Com relação à percepção sobre próprio esforço e dedicação, observou-se que em sua maioria os estudantes acreditam que dedicaram tempo suficiente para as tarefas $(66,70 \%)$. Entretanto, quando questionados sobre a qualidade das respostas, $46,70 \%$ entenderam que as respostas poderiam ser melhores. A grande maioria dos estudantes indicou que utilizou a ajuda sempre que acreditou que era necessário. Apenas 6,70\% indicou que não acessou, mas gostaria de tê-lo feito.
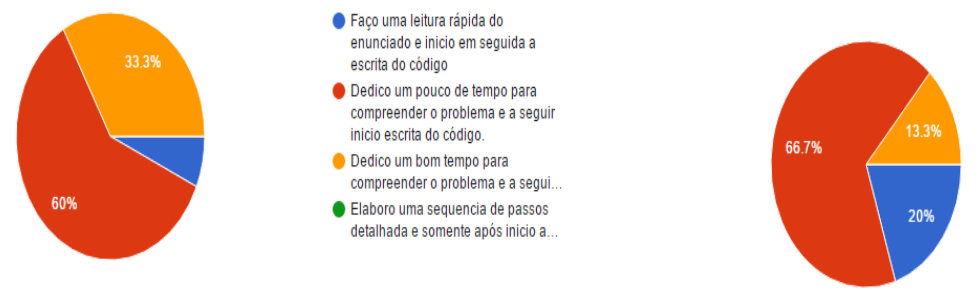

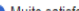

Satisfeito

Nem satisfeito, nem insatisfeito

Insatisfeito

Figura 3 - Respostas para as questões 12 e 13

A Figura 3 apresenta gráficos relacionados as respostas para as duas últimas questões. Quando questionados sobre como resolvem um problema na disciplina de algoritmos, mais da metade assinalou a alternativa que indicava "um pouco de tempo" e não "um bom tempo" para compreender o problema antes de escrever o código. Sobre a satisfação com as atividades realizadas durante o experimento, não houve respostas indicando insatisfação e $86 \%$ responderam que estavam muito satisfeitos ou satisfeitos.

\section{Considerações finais}

Este trabalho apresentou uma investigação sobre a problematização e a relação desta com fatores associados a motivação dos estudantes. Os resultados indicaram a viabilidade de utilizar os dados coletados para observar e identificar estes fatores.

Os tempos demandados em cada tarefa podem se constituir em bons indicativos para o reconhecimento do esforço dos discentes, entretanto, observou-se que tempos maiores nem sempre acarretam em respostas mais completas. A associação destes com os níveis de detalhe da descrição dos pontos chave e hipótese é necessária. Registros sobre acessos ao sistema e às dicas contribuem para identificar confiança e independência e a sua relação com os tempos, principalmente em situações de tempos fora do padrão pode indicar níveis mais altos ou baixos nestes fatores.

Em uma análise preliminar das respostas do relato dos estudantes, constatou-se que existem indicativos de que os alunos compreenderam a atividade e reconheceram a necessidade de refletir sobre os problemas. Os resultados permitem inferir que a abordagem pedagógica utilizada é promissora, porém, faz-se necessária ainda uma análise mais profunda sobre contribuição desta para o desenvolvimento das habilidades e competências associadas a programação.

Os resultados deste experimento servirão de base para o desenvolvimento de uma ferramenta de apoio para a aplicação da problematização e para o reconhecimento automático da motivação. Um sistema de apoio que contemple todas as etapas da atividade e que permita registrar detalhes sobre a elaboração do código fonte é importante para uma análise mais profunda dos tempos e das ações dos estudantes durante a resolução do exercício. Além do desenvolvimento da ferramenta, a continuidade das pesquisas, incluem a realização de novos experimentos, com o 
objetivo de obter mais dados e indicadores, e a aplicação de técnicas de mineração de dados para estabelecer relações entre as variáveis e para classificação automática dos níveis de dedicação, confiança e independência.

\section{Referências bibliográficas}

BENNEDSEN, Jens; CASPERSEN, Michael E. Failure rates in introductory programming. ACM SIGCSE Bulletin, v. 39, n. 2, p. 32-36, 2007.

BERBEL, Neusi Aparecida Navas; GAMBOA, Sílvio Ancízar Sánchez. A metodologia da problematização com o Arco de Maguerez: uma perspectiva teórica e epistemológica. Filosofia e Educação, v. 3, n. 2, p. 264-287, 2011.

BERBEL, Neusi Aparecida Navas. As metodologias ativas e a promoção da autonomia de estudantes. Semina: Ciências Sociais e Humanas, v.32, n.1, p.25-40, 2012.

BERCHT, M. Em Direção a Agentes Pedagógicos com Dimensões Afetivas. Tese de Doutorado. Instituto de Informática. UFRGS. 2001.

BORDENAVE, J. D.; PEREIRA, A. M. Estratégias de ensino-aprendizagem. 4. ed. Petrópolis: Vozes, 1982.

COCEA, Mihaela; WEIBELZAHL, Stephan. Disengagement detection in online learning: Validation studies and perspectives. Learning Technologies, IEEE Transactions on, v. 4, n. 2, p. 114-124, 2011.

DEL SOLATO, Teresa; DU BOULAY, Benedict. Implementation of motivational tactics in tutoring systems. Journal of Interactive Learning Research, v. 6, n. 4, p. 337, 1995.

DU BOULAY, Benedict; DEL SOLDATO, Teresa. Implementation of Motivational Tactics in Tutoring Systems: 20 years on. International Journal of Artificial Intelligence in Education, v. 26, n. 1, p. 170-182, 2016.

DA COSTA MORA, Michael; GIRAFFA, Lucia Maria Martins. Evasão na disciplina de algoritmo e programação: Um estudo a partir dos fatores intervenientes na perspectiva do aluno. In: Tercera Conferencia sobre el Abandono en la Educación Superior, III CLABES, 2013, Espanha.

IEPSEN, Edécio Fernando. Ensino de algoritmos: detecção do estado afetivo de frustração para apoio ao processo de aprendizagem. Tese de doutorado. 2013.

LONGHI, Magalí T.; BEHAR, Patricia A.; BERCHT, Magda. Máquina de inferência baseada na teoria bayesiana para identificar os estados de ânimo do aluno em um ambiente virtual de aprendizagem. RENOTE, v. 8, n. 3, 2010.

MEC: MINISTÉRIO DA EDUCAÇÃO. Diretrizes Curriculares Nacionais para os cursos de graduação na área da Computação. Brasília. 2016.

PICARD, Rosalind W. Affective computing. Cambridge: MIT Press, 1997.

RAMOS, Vinicius et al. A Comparação da Realidade Mundial do Ensino de Programação para Iniciantes com a Realidade Nacional: Revisão sistemática da literatura em eventos brasileiros. In: Brazilian Symposium on Computers in Education (Simpósio Brasileiro de Informática na Educação-SBIE). 2015. p. 318.

WATSON, Christopher; LI, Frederick WB. Failure rates in introductory programming revisited. In: Proceedings of the 2014 conference on Innovation \& technology in computer science education. ACM, 2014. p. 39-44. 